\title{
Diet and Chronic Inflammation ${ }^{1}$
}

\author{
Daniela Rivero-Mendoza and Wendy Dahl²
}

Inflammation is your body's response to injury and infection-it's how your immune system helps to protect you from harm. In contrast, chronic inflammation contributes to many diseases, including heart disease, diabetes, and kidney and liver disease. This publication discusses inflammation and the dietary choices that may help to reduce chronic inflammation.

\section{Inflammation}

Inflammation is part of the complex response of our body to harmful stimuli, such as bacteria, viruses, toxins, or even our own damaged cells. Through inflammation, the immune system can help eliminate disease-causing organisms and substances and promote tissue repair and recovery after an injury. Acute inflammation occurs when your body detects a harmful stimulus or damaged body tissue and reacts by sending out immune cells and substances around the affected area to help you heal. The site of inflammation, such as a cut or burn, becomes red, swollen, and warm-signs of inflammation that disappear with healing. In contrast, chronic inflammation occurs when the immune system continues to be activated, fighting too much and for too long. Chronic inflammation contributes to heart disease, diabetes, chronic kidney disease, non-alcoholic fatty liver disease, dementia, and autoimmune and movement disorders, among others (Furman et al. 2019).

\section{How to Measure Inflammation}

Your healthcare provider may test your blood for markers of inflammation; a common test is C-reactive protein (CRP). CRP is used to assess the severity of inflammation during illness or chronic disease. High-sensitivity Creactive protein (hs-CRP) is used to test for risk of cardiovascular disease (Li et al. 2017). Other common markers of inflammation include the interleukin (IL) family (e.g., IL-6) and tumor necrosis factor alpha (TNF- $\alpha$ ). Although CRP is commonly used by physicians to determine severity of disease, other inflammatory markers are more often measured in scientific studies.

\section{The American Diet and Chronic Inflammation}

The typical American diet is characterized by a low intake of fruits, vegetables, and fiber, and a high intake of refined grains and processed meats (USDHHS and USDA 2015). This diet is particularly high in ultraprocessed foods-foods that are highly processed with additives (Martínez Steele et al. 2016). This dietary pattern is thought to alter the gut microbiota composition, intestinal environment, and immune system, which ultimately leads to chronic inflammation (Furman et al. 2019). Overconsumption of ultraprocessed foods in particular may also promote obesity, which further provokes chronic inflammation (Spyridaki, Avgoustinaki, and Margioris 2016). By improving diet quality and maintaining a healthy weight, risk of chronic inflammation may be decreased.

1. This document is FSHN20-43, one of a series of the Food Science and Human Nutrition Department, UF/IFAS Extension. Original publication date September 2020. Visit the EDIS website at https://edis.ifas.ufl.edu for the currently supported version of this publication.

2. Daniela Rivero-Mendoza, Extension and research coordinator; and Wendy Dahl, associate professor, Food Science and Human Nutrition Department; UF/IFAS Extension; Gainesville, FL 32611.

The Institute of Food and Agricultural Sciences (IFAS) is an Equal Opportunity Institution authorized to provide research, educational information and other services

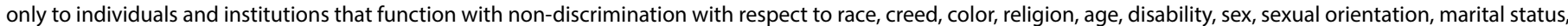

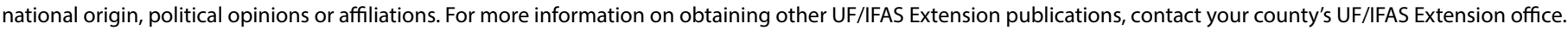
U.S. Department of Agriculture, UF/IFAS Extension Service, University of Florida, IFAS, Florida A \& M University Cooperative Extension Program, and Boards of County Commissioners Cooperating. Nick T. Place, dean for UF/IFAS Extension. 
While a low-quality diet promotes chronic inflammation, some dietary patterns and foods may help with prevention of diseases related to chronic inflammation.

\section{Diet and Chronic Inflammation}

Fruits and vegetables contain vitamins, minerals, fiber, and phytonutrients (beneficial substances in plants) that promote health and may help to prevent chronic inflammation. A higher intake of fruits and vegetables leads to a decrease in inflammatory markers, specifically CRP and TNF- $\alpha$ (Hosseini et al. 2018). The Dietary Guidelines for Americans provides guidance on the types and amounts of fruits and vegetables for good health (USDHHS and USDA 2015). Choosing more fruits and vegetables may help to avoid undesired chronic inflammation.

Whole grains contain fiber, vitamins, minerals, and phytonutrients that promote health. Although some studies show that higher intakes of whole grains are associated with decreased inflammation, other studies do not confirm this relationship. However, in unhealthy individuals, markers of inflammation improve with higher intakes of whole grains (Rahmani et al. 2020). The Dietary Guidelines for Americans recommends choosing whole grains to promote health (USDHHS and USDA 2015).

Dairy foods provide an important source of protein, calcium, and other nutrients, and may help to decrease chronic inflammation. Research supports that higher consumption of dairy foods compared with low or no intake, led to reduced inflammatory markers including CRP, TNF- $\alpha$, and IL-6 (Moosavian et al. 2020).

Replacing solid fats with healthy oils is recommended in the Dietary Guidelines for Americans (USDHHS and USDA 2015). Olive oil intake has been shown to decrease inflammatory markers, specifically CRP and IL-6 (Schwingshackl, Christoph, and Hoffmann 2015). However, the evidence for omega-3 fats, found in fish and flaxseed, for improving inflammation is less certain. In a review of studies that tested the effects of omega- 3 intake on inflammatory markers, no clear benefits of omega- 3 were identified (Natto et al. 2019).

\section{Dietary Patterns and Chronic Inflammation}

Food synergy is the idea that more benefit can be obtained by healthful dietary patterns, rather than from single foods or nutrients (Jacobs, Gross, and Tapsell 2009). Some plant-based diets, given their combination of healthful foods, seem to be promising for decreasing inflammation.

A plant-based dietary pattern emphasizes whole grains, fruits, vegetables, legumes, nuts, and plant-sourced oils, and may or may not include animal products. Plant-based dietary patterns include the Mediterranean diets, Dietary Approaches to Stop Hypertension (DASH), and various forms of a vegetarian diet, among others. As a whole, plantbased foods have been shown to be associated with overall improvement in inflammatory profiles when compared with other dietary patterns (Eichelmann et al. 2016).

A vegetarian dietary pattern excludes meat, poultry, and seafood, and may or may not include products derived from animals such as dairy and eggs. Healthful vegetarian diets were associated with decreased inflammatory markers when compared to a well-balanced mixed diet, a diet for type 2 diabetes, various habitual mixed diets, and a diet based on the American Heart Association guidelines (Craddock et al. 2019). However, more studies are needed to confirm whether vegetarian diets are protective against chronic inflammation. If you are a vegetarian, or you are planning to become one, talk to a registered dietitian or other health care provider to make sure your diet choices meet your nutrient needs. A healthful vegetarian dietary pattern may help to reduce inflammation.

The DASH diet was designed to improve blood pressure and has also been shown to help prevent cardiovascular disease (Siervo et al. 2015). The diet promotes fruits, vegetables, and low-fat and fat-free dairy products, as well as whole grains, poultry, fish, nuts, seeds, and legumes, while restricting refined grains, sugary beverages, saturated fats, and red meats. The DASH diet is low in saturated fat and sodium and promotes foods higher in potassium, calcium, magnesium, and fiber. The DASH diet decreases the inflammatory marker hs-CRP, compared to usual diets, but was no better than other healthy dietary patterns (Soltani, Chitsazi, and Salehi-Abargouei 2018). Consuming a healthy diet, such as the DASH diet, may help to decrease inflammatory markers.

The Mediterranean dietary pattern promotes virgin olive oil, vegetables, fruits, whole grains, fish, a moderate intake of poultry, eggs, dairy and alcohol, and low red meat, sweets, and pastry consumption. Several studies have studied the effects of a Mediterranean dietary pattern on inflammatory markers. A review of these studies found that the Mediterranean dietary pattern improved several markers of inflammation (e.g. hs-CRP, IL-6) (Schwingshackl and Hoffmann 2014). Overall, the Mediterranean 
dietary pattern is a good option to promote health and may decrease chronic inflammation.

A low glycemic diet is a dietary pattern that focuses on choosing low glycemic index (GI) foods and foods with a lower glycemic load (GL). Glycemic index is not only important for the control of blood glucose for those living with diabetes but may also play a role in decreasing inflammation (Schwingshackl and Hoffmann 2013). A review of studies has shown that decreases in the inflammatory marker CRP were more pronounced in adults who consumed lower GI/GL, compared to those who consumed higher GI/GL foods, even when studies including adults with type 2 diabetes were excluded (Schwingshackl and Hoffmann 2013). Adopting long-term, low glycemic diets may help to decrease inflammation.

Glycemic Index (GI) is a way of ranking foods based on their effects on blood glucose in relation to a reference food.

Glycemic load (GL) takes into account the amount of available carbohydrate in a serving of food. GL is calculated by multiplying the Gl of a particular food by the amount of "available" carbohydrate in a serving of the food and dividing this factor by 100. "Available" carbohydrate is that which is digested and absorbed as sugar (e.g. total carbohydrate in the food minus the fiber). Foods with lower $\mathrm{Gl}$, such as legumes, slowly release glucose into the bloodstream, whereas foods with higher $\mathrm{Gl}$, such as bread and most breakfast cereals, release glucose rapidly. The Gl of carrots is high, but when considering the low carbohydrate content of carrots, they have a low GL. Considering both the $\mathrm{Gl}$ and $\mathrm{GL}$ is important when choosing a low glycemic diet.

\section{Conclusion}

Inflammation helps you heal, whereas chronic inflammation contributes to chronic disease. Healthful dietary patterns and food choice may help to reduce chronic inflammation, whereas the typical American diet and obesity promote inflammation. Healthful plant-based dietary patterns, with an emphasis on fruits, vegetables, whole grains, legumes, nuts, and seeds may help to reduce chronic inflammation.

\section{References}

Craddock, J. C., E. P. Neale, G. E. Peoples, and Y. C. Probst. 2019. "Vegetarian-Based Dietary Patterns and Their Relation with Inflammatory and Immune Biomarkers: A Systematic Review and Meta-Analysis." Advances in Nutrition 10 (3): 433-451. https://doi.org/10.1093/advances/ nmy103
Eichelmann, F., L. Schwingshackl, V. Fedirko, and K. Aleksandrova. 2016. "Effect of Plant-Based Diets on Obesity-Related Inflammatory Profiles: A Systematic Review and Meta-analysis of Intervention Trials." Obesity Reviews 17 (11): 1067-1079. https://doi.org/10.1111/obr.12439

Furman, David, Judith Campisi, Eric Verdin, Pedro Carrera-Bastos, Sasha Targ, Claudio Franceschi, Luigi Ferrucci, Derek W. Gilroy, Alessio Fasano, Gary W. Miller, Andrew H. Miller, Alberto Mantovani, Cornelia M. Weyand, Nir Barzilai, Jorg J. Goronzy, Thomas A. Rando, Rita B. Effros, Alejandro Lucia, Nicole Kleinstreuer, and George M. Slavich. 2019. "Chronic Inflammation in the Etiology of Disease across the Life Span." Nature Medicine 25 (12): 1822-1832. https://doi.org/10.1038/s41591-019-0675-0

Hosseini, Banafshe, Bronwyn S. Berthon, Ahmad Saedisomeolia, Malcolm R. Starkey, Adam Collison, Peter A. B. Wark, and Lisa G. Wood. 2018. "Effects of Fruit and Vegetable Consumption on Inflammatory Biomarkers and Immune Cell Populations: A Systematic Literature Review and Meta-analysis." The American Journal of Clinical Nutrition 108 (1): 136-155. https://doi.org/10.1093/ajcn/nqy082

Jacobs, D. R., Jr., M. D. Gross, and L. C. Tapsell. 2009. "Food Synergy: An Operational Concept for Understanding Nutrition." American Journal of Clinical Nutrition 89 (5): 1543s-1548s. https://doi.org/10.3945/ajcn.2009.26736B

Li, Y., X. Zhong, G. Cheng, C. Zhao, L. Zhang, Y. Hong, Q. Wan, R. He, and Z. Wang. 2017. "Hs-CRP and All-Cause, Cardiovascular, and Cancer Mortality Risk: A Meta-analysis." Atherosclerosis 259:75-82. https://doi.org/10.1016/j. atherosclerosis.2017.02.003

Martínez Steele, Eurídice, Larissa Galastri Baraldi, Maria Laura da Costa Louzada, Jean-Claude Moubarac, Dariush Mozaffarian, and Carlos Augusto Monteiro. 2016. "Ultra-processed Foods and Added Sugars in the US Diet: Evidence from a Nationally Representative Cross-Sectional Study." BMJ Open 6 (3): e009892. https://doi.org/10.1136/ bmjopen-2015-009892

Moosavian, Seyedeh Parisa, Mehran Rahimlou, Parvane Saneei, and Ahmad Esmaillzadeh. 2020. "Effects of Dairy Products Consumption on Inflammatory Biomarkers among Adults: A Systematic Review and Meta-analysis of Randomized Controlled Trials." Nutrition, Metabolism and Cardiovascular Diseases. https://doi.org/10.1016/j. numecd.2020.01.011 
Natto, Zuhair S., Wael Yaghmoor, Heba K. Alshaeri, and Thomas E. Van Dyke. 2019. "Omega-3 Fatty Acids Effects on Inflammatory Biomarkers and Lipid Profiles among Diabetic and Cardiovascular Disease Patients: A Systematic Review and Meta-analysis." Scientific Reports 9 (1): 18867. https://doi.org/10.1038/s41598-019-54535-x

Rahmani, Sepideh, Omid Sadeghi, Mehdi Sadeghian, Narges Sadeghi, Bagher Larijani, and Ahmad Esmaillzadeh. 2020. "The Effect of Whole-Grain Intake on Biomarkers of Subclinical Inflammation: A Comprehensive Meta-analysis of Randomized Controlled Trials." Advances in Nutrition 11 (1): 52-65. https://doi.org/10.1093/advances/nmz063

Schwingshackl, L., and G. Hoffmann. 2013. "Long-Term Effects of Low Glycemic Index/Load vs. High Glycemic Index/Load Diets on Parameters of Obesity and ObesityAssociated Risks: A Systematic Review and Meta-analysis." Nutrition, Metabolism and Cardiovascular Diseases 23 (8): 699-706. https://doi.org/10.1016/j.numecd.2013.04.008

Schwingshackl, L., and G. Hoffmann. 2014. "Mediterranean Dietary Pattern, Inflammation and Endothelial Function: A Systematic Review and Meta-analysis of Intervention Trials." Nutrition, Metabolism and Cardiovascular Diseases 24 (9): 929-939. https://doi.org/10.1016/j.numecd.2014.03.003

Schwingshackl, Lukas, Marina Christoph, and Georg Hoffmann. 2015. "Effects of Olive Oil on Markers of Inflammation and Endothelial Function-A Systematic Review and Meta-analysis." Nutrients 7 (9): 7651-7675. https://doi.org/10.3390/nu7095356

Siervo, M., J. Lara, S. Chowdhury, A. Ashor, C. Oggioni, and J. C. Mathers. 2015. "Effects of the Dietary Approach to Stop Hypertension (DASH) Diet on Cardiovascular Risk Factors: A Systematic Review and Meta-analysis." Br J Nutr 113 (1): 1-15. https://doi.org/10.1017/s0007114514003341

Soltani, Sepideh, Maryam J. Chitsazi, and Amin SalehiAbargouei. 2018. "The Effect of Dietary Approaches to Stop Hypertension (Dash) on Serum Inflammatory Markers: A Systematic Review and Meta-analysis of Randomized Trials." Clinical Nutrition 37 (2): 542-550. https://doi. org/10.1016/j.clnu.2017.02.018
Spyridaki, Eirini C., Pavlina D. Avgoustinaki, and Andrew N. Margioris. 2016. "Obesity, Inflammation and Cognition." Current Opinion in Behavioral Sciences 9:169-175. https:// doi.org/10.1016/j.cobeha.2016.05.004

USDHHS and USDA. 2015. "2015 - 2020 Dietary Guidelines for Americans. 8th Edition." http://health.gov/ dietaryguidelines/2015/guidelines/ 